\title{
El rol de las capacidades dinámicas de networking en la internacionalización de pymes latinoamericanas
}

\section{The role of dynamic networking capabilities in the internationalization of Latin American} SMES

\section{Nidia Roa Vivas ${ }^{1}$ \\ Paula Andrea Garcia Ortiz ${ }^{2}$ \\ Haydée Calderón Garcia ${ }^{3}$ \\ Teresa Fayos Gardó 4}

\begin{abstract}
(2)
\end{abstract}
Recepción: 27/08/2020
(.).

Aprobación: 25/09/2020
Publicación: 18/12/2020

\section{Para citar este artículo:}

Roa Vivas, N., García Ortiz, P.A., Calderón García, H., \& Fayos Gardó,

T. (2020). El rol de las capacidades dinámicas de networking en la internacionalización de las pymes latinoamericanas. Indagare, (8), 171-177. https://doi.org/10.35707/indagare/814

\section{(9) $(\Theta \Theta \Theta$}

\footnotetext{
${ }^{1}$ Grupo de investigación UnIDERE, Universidad de Ibagué, Colombia. ORCID: 0000-0003-4664-6627. Correo electrónico: nidia.roa@unibague.edu.co

${ }^{2}$ Grupo de investigación UNIDERE, Universidad de Ibagué, Colombia. ORCID: 0000-0002-4262-0211. Correo electrónico: paula.garcia@unibague.edu.co

${ }^{3}$ Grupo de investigación CANALDI, Universidad de Valencia, España. ORCID: 0000-0002-4774-7465. Correo electrónico: haydee.calderon@uv.es

${ }^{4}$ Grupo de investigación CANALDI, Universidad de Valencia, España. ORCID: 0000-0003-3742-7844. Correo electrónico: teresa.fayos@uv.es
} 


\title{
Resumen
}

Las pymes latinoamericanas difícilmente evidencian experiencias de internacionalización significativas debido a sus limitaciones de conocimiento, recursos y relaciones con intermediarios estratégicos. El reto es generar cooperación entre todos los partners y orquestar la combinación adecuada que permita integrar el canal de distribución internacional. El objetivo de esta investigación ha sido identificar aquellas capacidades dinámicas específicas de networking necesarias para el desarrollo de la estrategia de distribución, y así, impulsar los procesos de internacionalización de pymes latinoamericanas. Los resultados demuestran que los gerentes identifican, desarrollan $\mathrm{y}$ fortalecen sus relaciones con distribuidores y agentes internacionales, que finalmente les permiten dinamizar su proceso de internacionalización y superar sus rigideces organizacionales.

\begin{abstract}
Latin American SMEs hardly evince significant internationalization experiences due to their limited knowledge, resources, and relationships with strategic intermediaries. The challenge is to generate cooperation between all partners and conduct the appropriate combination that allows integrating the international distribution channel. The aim of this research has been to identify those specific dynamic networking capabilities necessary for the development of the distribution strategy, and thus, promote the internationalization processes of Latin American SMEs. The results show that managers identify, develop and strengthen their relationships with international distributors and agents, which ultimately allow them to enliven their internationalization process and overcome their organizational severity.
\end{abstract}

\footnotetext{
Palabras claves: Networking, capacidades dinámicas de marketing, canal de distribución.

Key words: Networking, dynamic marketing capabilities, distribution channel.
}

\section{Introducción}

Las capacidades dinámicas determinan la velocidad y el nivel en que los recursos relevantes pueden alinearse y ajustarse. La alineación de los recursos internos y externos indica en qué momento y cómo la organización precisa establecer redes que involucran otras 
INDAGA3E e-ISSN: 2357-5042 • Número 8 (2020) • Universidad de Ibagué • doi: https://doi.org/10.35707/indagare/814

empresas (Carattoli, Annunzio \& Dupleix, 2014). Las redes de negocio se definen como aquellas que mantienen las organizaciones con clientes, distribuidores, competidores y gobierno. En el grado en que las empresas se internacionalizan, el número de actores con quienes interactúan en la red aumenta y las relaciones con estos se convierten en más cercanas (Oviatt \& McDougall, 1994).

El carácter relevante de las capacidades dinámicas en la sostenibilidad de multinacionales y sus conexiones es ampliamente reconocido (Evers, 2011; Frasquet, Dawson \& Mollá, 2013). Sin embargo, para Fletcher \& Harris (2012), el enfoque reside en las pequeñas y medianas empresas que difícilmente ostentan una experiencia significativa para desarrollar procesos de internacionalización más avanzados en mercados como el latinoamericano. Esto se presenta debido a la falta de redes y a la ausencia de la construcción de conocimiento. La experiencia significativa solo puede lograrse con networking para la internacionalización temprana inicial, basada en intermediarios que ayudan a conectar huecos estructurales y proporcionan heterogeneidad en la red para acceder a más experiencias de aprendizaje (Musteen, Datta \& Butts, 2013). Además, los intermediarios aportan los recursos VRIO (Valiosos, raros, inimitables e importantes para la organización) fundamentales para los canales de venta (Watson, Worm, Palmatier \& Ganesan, 2015).

Figura 1. Estancia de docencia e investigación - Universidad de Lima (Perú) - Docente Nidia Roa Vivas - Alianza del Pacífico (2018)

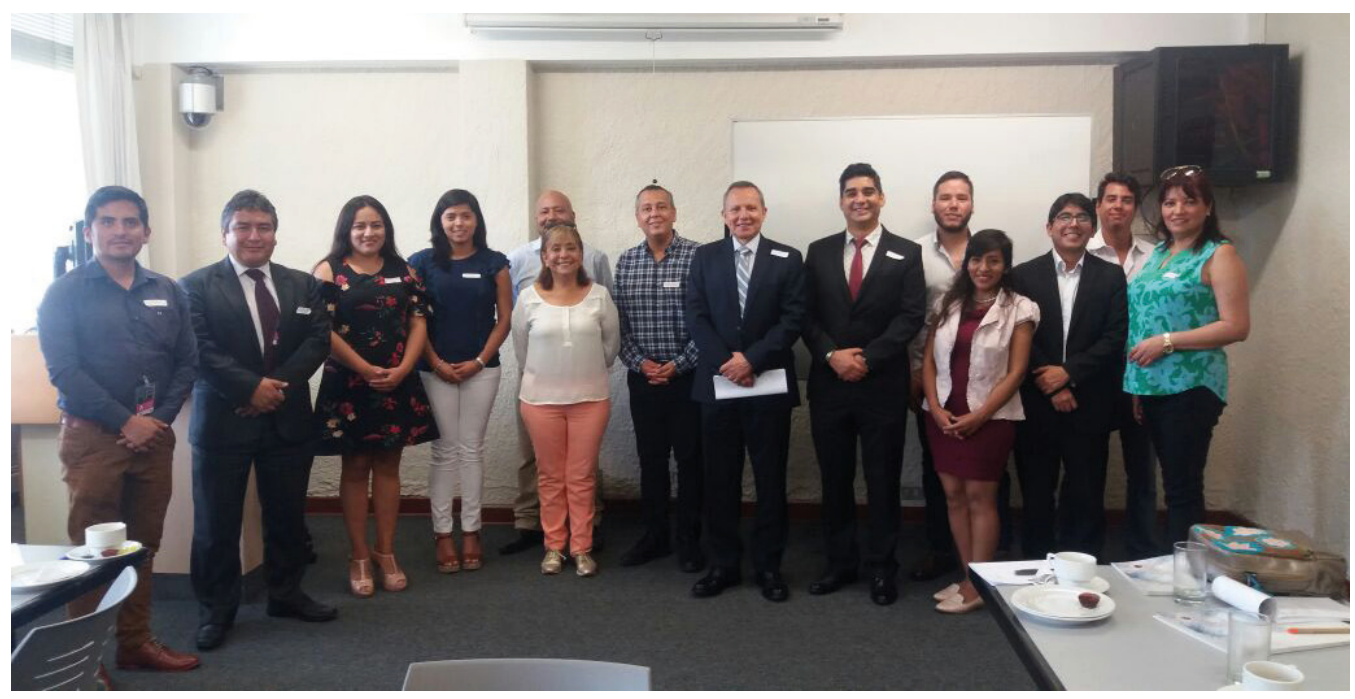

Fuente: Universidad de Lima (2018) 
Poco ha sido el estudio realizado sobre el impacto de las capacidades de networking en la internacionalización (Bai \& Johanson, 2018). Autores como Watson et al. (2015) sugieren enfocar ambos elementos hacia la estrategia de distribución, que permite hacer frente a diversas dificultades propias de las pymes como restricciones de recursos y tiempo (O’Toole \& McGrath, 2018). Pese a que diversos estudios han sido enfocados en las oportunidades de lograr interacción y sinergia de los canales, la importancia de cómo organizaciones específicas pueden construir nuevas capacidades dinámicas al explotar las sinergias e interacciones a lo largo de los diferentes canales de distribución sigue sin ser analizada (D’atri, De Marco, Braccini \& Cabiddu, 2010; Kumar, Sunder \& Sharma, 2015; Neslin et al., 2006).

En consecuencia, nuestro objetivo es identificar aquellas capacidades dinámicas de networking que permiten a las pymes explotar sus relaciones actuales y explorar nuevas relaciones con sus intermediarios para mejorar sus procesos de internacionalización. De acuerdo con este propósito principal hacemos tres preguntas fundamentales: 1) ¿la capacidad dinámica específica de identificar, desarrollar y fortalecer relaciones favorece que las empresas desarrollen su canal de distribución? Este desarrollo ¿facilita la internacionalización de la pyme?, 2) ¿la capacidad dinámica específica de acceder, movilizar y coordinar recursos favorece que las empresas desarrollen múltiples canales? Este desarrollo ¿facilita la internacionalización de la pyme? y 3) ¿la capacidad dinámica específica de recibir, absorber e interpretar información y conocimiento favorece que las empresas desarrollen múltiples canales? Este desarrollo ¿facilita la internacionalización de la pyme?

Para abordar estas cuestiones, realizamos una amplia revisión de la literatura sobre las capacidades dinámicas de networking y canales de distribución. A continuación, exploramos el impacto de estas capacidades en las diferentes funciones de los canales de distribución de las empresas latinoamericanas que se encuentran en las primeras etapas del proceso de internacionalización. Por último, esbozamos los materiales y métodos, resultados y fotografías de ejecución del proyecto.

\section{Materiales y métodos}

Hemos aplicado una metodología de recolección de la información cualitativa, a través del análisis de casos múltiples de quince empresas colombianas y peruanas internacionalizadas. Como indican Cassell (2015) y Yin (2014) este diseño es ampliamente utilizado cuando el fenómeno se está formando con el objetivo de generar rich data. En este caso se ha desarrollado bajo el enfoque progresivo de Sinkovics \& Alfoldi (2012), y se ha utilizado el software CAQDAS Atlas ti 7.5.15 para documentar todo el proceso de investigación. 


\section{Resultados}

Se pudo detectar que los gerentes identifican, desarrollan y fortalecen relaciones estratégicas con agentes y distribuidores, para dinamizar sus ventas internacionales. De esta manera generan una comunicación y colaboración sinérgica de la información sobre productos, soporte técnico y acompañamiento para sus aliados estratégicos, que favorece su internacionalización.

De hecho, existe una comunicación intensa entre las empresas productoras y los distribuidores, para coordinar la información disponible en portales web sobre las características de los productos, así facilitar el contacto con los clientes extranjeros. Esta coordinación es soportada por el flujo de recursos entre los miembros del canal, dichos recursos se exhiben en capacitación continua con los distribuidores (usos del producto, características técnicas, componentes) a través de plataformas digitales y flujo de material para el punto de venta, junto con muestras gratis para favorecer el proceso de venta en el país extranjero.

Figura 2. Estancia de investigación - Docente Paula Andrea García - Beca Jóvenes Investigadores 2019 del Programa de Cooperación de la Universitat de València,Universitat De València

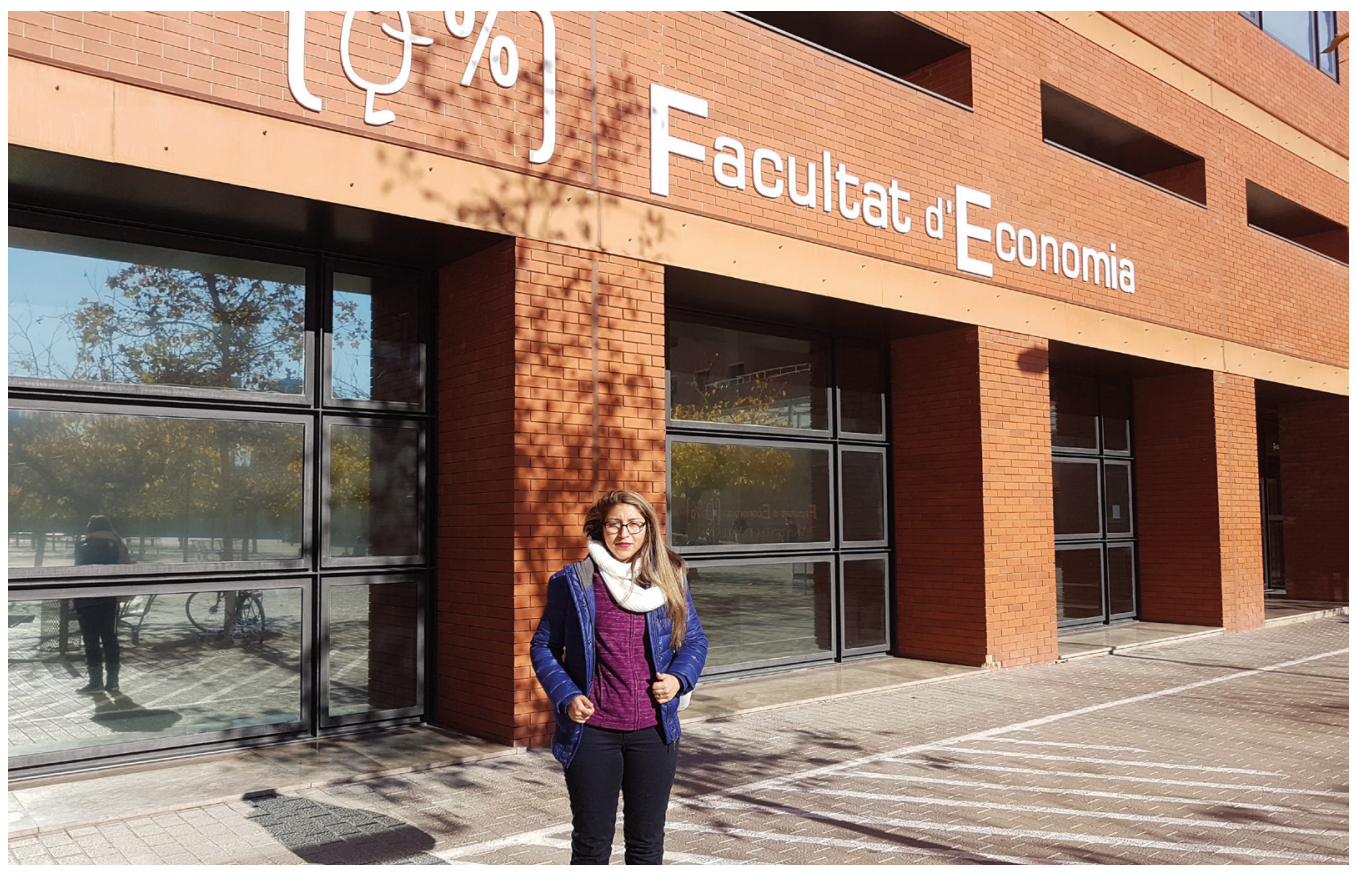

Fuente: Universidad de Valencia (2019) 
INDAGAZEE e-ISSN: 2357-5042 • Número 8 (2020) • Universidad de Ibagué • doi: https://doi.org/10.35707/indagare/814

\section{Potencial uso}

El modelo conceptual ofrece a los gerentes la posibilidad de identificar capacidades específicas de networking que ayuden a una empresa latinoamericana a impulsar su proceso de internacionalización y a establecer operaciones y decisiones sincronizadas con sus intermediarios internacionales. Por lo tanto, estos hallazgos son valiosos para los empresarios e investigadores que desean saber qué tipos de capacidades de networking son apropiadas y cómo construirlas, mantenerlas y mejorarlas en las empresas latinoamericanas, para integrar las funciones del canal internacional y desplegar procesos de internacionalización más avanzados.

\section{Ficha técnica del proyecto}

Título del proyecto: Capacidades dinámicas de networking en la internacionalización de empresas en países en proceso de desarrollo en América Latina: especial referencia a las empresas colombianas.

PRIT: Industria de servicios de alto valor agregado y de talla internacional.

Código del proyecto: $18-569$-INT.

Palabras clave: Capacidades dinámicas, networking, canal de distribución, internacionalización.

Grupo de investigación: UNIDERE.

Investigador principal: Nidia Roa Vivas.

Correo electrónico: nidia.roa@unibague.edu.co

\section{Referencias}

Bai, W., \& Johanson, M. (2018). International opportunity networks. Industrial Marketing Management, 70, 167-179. https://doi.org/10.1016/j.indmarman.2017.07.004

Carattoli, M., D’Annunzio, C., \& Dupleix, D. (2014). Proceso de desarrollo de capacidades dinámicas en pequeñas y medianas empresas de software. Pymes, Innovación y Desarrollo, 2(1), 1-30. Recuperado de https://revistas.unc.edu.ar/index.php/pid/article/view/7558

Cassell, C. (2015) Qualitative research methods. Oxford bibliographies in management. New York: Oxford University Press.

D'atri, A., De Marco, M., Braccini, A. M., \& Cabiddu, F. (Eds.). (2010). Management of the Interconnected World: ItAIS: The Italian Association for Information Systems. Naples: Italy: Springer Science \& Business Media.

Evers, N. (2011). International new ventures in "low tech" sectors: a dynamic capabilities perspective. Journal of Small Business and Enterprise Development, 18(3), 502-528. https://doi.org/10.1108/14626001111155682 
INDAGA3E e-ISSN: 2357-5042 • Número 8 (2020) • Universidad de Ibagué • doi: https://doi.org/10.35707/indagare/814

Fletcher, M., \& Harris, S. (2012). Knowledge acquisition for the internationalization of the smaller firm: Content and sources. International Business Review, 21(4), 631-647. https://doi.org/10.1016/j. ibusrev.2011.07.008

Frasquet, M., Dawson, J., \& Mollá, A. (2013). Post-entry internationalisation activity of retailers: An assessment of dynamic capabilities. Management Decision, 51(7), 1510-1527. https://doi.org/10.1108/ MD-02-2013-0081

Kumar, V., Sunder, S., \& Sharma, A. (2015). Leveraging distribution to maximize firm performance in emerging markets. Journal of Retailing, 91(4), 627-643. https://doi.org/10.1016/j.jretai.2014.08.005

Musteen, M., Datta, D. K., \& Butts, M. M. (2014). Do international networks and foreign market knowledge facilitate SME internationalization? Evidence from the Czech Republic. Entrepreneurship Theory and Practice, 38(4), 749-774. https://doi.org/10.1111/etap.12025

Neslin, S., Grewal, D., Leghorn, R., Shankar, V., Teerling, M., Thomas, J., \& Verhoef, P. (2006). Challenges and opportunities in multichannel customer management. Journal of Service Research, 9(2), 95-112. https://doi.org/10.1177/1094670506293559

O' Toole, T., \& McGrath, H. (2018). Strategic patterns in the development of network capability in new ventures. Industrial Marketing Management, 70, 128-140. https://doi.org/10.1016/j. indmarman.2017.07.003

Oviatt, B. M., \& McDougall, P. P. (1994). Toward a theory of international new ventures. Journal of international business studies, 25(1), 45-64. https://doi.org/10.1057/palgrave.jibs.8490193

Sinkovics, R. R., \& Alfoldi, E. A. (2012). Progressive Focusing and Trustworthiness in Qualitative Research: The Enabling Role of Computer-Assisted Qualitative Data Analysis Software (CAQDAS). Management International Review, 52(6), 817-845. https://doi.org/10.1007/s11575-012-0140-5

Watson, G. F., Worm, S., Palmatier, R. W., \& Ganesan, S. (2015). The evolution of marketing channels: trends and research directions. Journal of Retailing, 91(4), 546-568. https://doi.org/10.1016/j. jretai.2015.04.002

Yin, R. (2014). Case study research and applications: Design and methods. London, England: Sage publications. 